\title{
Evaluation of Functional Outcome of Cemented Hip Replacements
}

Hossain $\mathrm{T}^{1^{*}}$, Rahman $\mathrm{MH}^{2}$, Kader $\mathrm{A}^{3}$, Alam $\mathrm{SMD}^{4}$

\author{
${ }^{1}$ Dr. Md. Tofayel Hossain, Associate Professor, Department of Orthopaedic Surgery, National Institute of Traumatology \\ and Orthopedic Rehabilitation (NITOR), Dhaka, Bangladesh \\ ${ }^{2}$ Dr. Mir Hamidur Rahman, Associate Professor, Department of Orthopaedic Surgery, National Institute of Traumatology \\ and Orthopedic Rehabilitation (NITOR), Dhaka, Bangladesh \\ ${ }^{3}$ Dr. Md Abdul Kader, Assistant Professor, Department of Orthopaedic Surgery, Khulna Medical College, Khulna, \\ Bangladesh \\ ${ }^{4}$ Dr. SM Didarul Alam, Sr. Consultants, Department of Orthopaedic Surgery, Khulna Medical College, Khulna, Bangladesh
}

DOI: $10.36347 /$ sjams.2020.v08i12.034

| Received: 06.12.2020 | Accepted: 17.12.2020 | Published: 29.12.2020

*Corresponding author: Dr. Md. Tofayel Hossain

\section{Abstract}

Original Research Article

Introduction: This article is to observe the functional outcome of cemented hip replacements. A cemented joint prosthesis uses fast-drying bone cement to help affix it to the bone. A cementless joint prosthesis, sometimes called a pressfit prosthesis, is specially textured to allow the bone to grow onto it and adhere to it over time. This article is focusedon the functional outcome of a cemented hip replacement and any changes after surgery. The main component of a cemented hip replacement is Polymethylmethacrylate, more commonly known as (PMMA). (PMMA) is a kind of bone cement technology that originated from industrial Plexiglass administration in the 1950s to the recent advent of nanoparticle additives. Various types of additives have been trialed to figure out problems with modern bone cements, like loosening of the prosthesis, high post-operative infection rates, and inflammatory reduction in interface integrity.1Proximal femur undergoes physical changes to carry the weight of the body. Thus, any affection to the hip joint severely affects locomotion [2]. This study focused on the outcome of a successful cemented THR surgery and its possible complications. It also showed the male to female ratio in cases of hip replacements, based on our current socio-economic status, and the average age of a patient going in for a cemented hip replacement. Aim of the study: This study aimed to evaluate the functional outcome of Cemented hip replacement of post-surgery. Methodology: This was an observational study and was conducted in the Department of Orthopaedic Surgery, of National Institute of Traumatology and Orthopedic Rehabilitation (NITOR), Dhaka, Bangladesh during the period from April 2018 to March 2020, over a period of two years. Records of patients with cemented hip replacements were collected over the two-year period, by giving them a questionnaire at their follow up inspection, which happened 5-10 years after the surgery. The questionnaire was given to50 different patients, all with cemented hip arthroplasty done before, including a wide range of demographics. Of the 50 initial prospects, 2 patients did not wish to disclose their personal information, bringing the sample number to 48 willing to participate in this study. The collected data was then cross-referenced with similar existing case studies to reach an outcome. Result: A total of 58.33\% ( $\mathrm{n}=28)$ patients were under the age of 55 at the time of surgery, $16.67 \%(n=8)$ were between the age group of 55 to $65.18 .75 \%(n=9)$ patients were from the age group of 66 to 75 years, and the rest $6.250 \%(n=3)$ were above 75 at the time of their arthroplasty. It is to be noted that this data was heavily influenced by the average mortality rate of our country as our average life expectancy is 72.05 years. At the time of the follow-up, only $91.67 \%(n=44)$ were alive, and the remaining $8.33 \%(n=4)$ were found to have passed away, of which, $6.25 \%(n=3)$ had departed of natural causes, and $2.08 \%(n=1)$ died of surgery complications. The follow-up year after their initial surgery was divided into 5 groups, starting at 6 years after their surgery having a $15.91 \%(n=7)$ patients coming for secondary revision, $11.36 \%(n=5)$ patients coming 7 years after the surgery, $13.64 \%(n=6)$ having followed up 8 years after the surgery, $27.27 \%(n=12)$ having followed up 9 years after the THA and the majority having done the follow up 10 years after successful surgery, at $31.82 \%(n=14)$. The primary reasons for a follow-up can be divided into 3 primary groups, having the majority of patients getting follow up for resurfacing pain, at $72.73 \%(n=32), 13.64 \%(n=6)$ for sepsis, and 13.64\% $(n=6)$ coming in for dislocation. Conclusion: For example $95.9 \%$ of cemented implants survived a 10 ears in patients aged $\geq 75$ years compared with $93 \%$ of uncemented, $93.9 \%$ of hybrid and $93.2 \%$ of reverce hybrid. The survival of cemented replacements in patients aged 55 to 64 years was similar.

Keywords: THR, Arthroplasty, Hip replacement, cemented, cementless, follow-up, THA.

Copyright $\odot 2020$ The Author(s): This is an open-access article distributed under the terms of the Creative Commons Attribution 4.0 International License (CC BY-NC 4.0) which permits unrestricted use, distribution, and reproduction in any medium for non-commercial use provided the original author and source are credited. 


\section{INTRODUCTION}

When a patient suffering from chronic joint pain due to arthritis opts to have joint replacement surgery, the orthopedic surgeon will replace the existing joint surfaces with artificial joint prostheses. These prosthetic components must adhere to the patient's natural bone. This adhesion can be achieved in two ways. A cemented replacement and a noncemented replacement. A cemented joint prosthesis uses fastdrying bone cement to help affix it to the bone. A cementless joint prosthesis, sometimes called a press-fit prosthesis, is specially textured to allow the bone to grow onto it and adhere to it over time. This article is focusedon the functional outcome of a cemented hip replacement and any changes after surgery. The main component of a cemented hip replacement is Polymethylmethacrylate, more commonly known as (PMMA). (PMMA) is a kind of bone cement technology that originated from industrial Plexiglass administration in the 1950 to the recent advent of nanoparticle additives. Various types of additives have been trialed to figure out problems with modern bone cements, like loosening of the prosthesis, high postoperative infection rates, and inflammatory reduction in interface integrity [1]. Proximal femur undergoes physical changes to carry the weight of the body. Thus, any affection to the hip joint severely affects locomotion [2]. This study focused on the outcome of a successful cemented THR surgery and its possible complications. It also showed the male to female ratio in cases of hip replacements, based on our current socio-economic status, and the average age of a patient going in for a cemented hip replacement. This study focused mainly on the outcome of a THR and evaluated them to reach proper conclusions. It has also examined the possible complications regarding these types of surgery, specifically the postoperative complications, to see if cemented total hip arthroplasty is a viable option for patients with severe pain in the coxa region. The study has also concluded the primary reasons for a follow up after a successful hip replacement, which weighed in on the types of possible complications faced in these types of major surgery.

\section{OBJECTIVES}

General objective: To evaluate the functional outcome of Cemented Hip Replacements.

Specific Objectives: To discuss the functional outcome after a successful THR, to observe the positive or negative influence in a patient's life after a cemented hip replacement.

\section{Methodology \& Materials}

This was an observational study and was conducted in the Department of Orthopaedic Surgery of National Institute of Traumatology and Orthopedic Rehabilitation (NITOR), Dhaka, Bangladesh during the period from April 2018 to March 2020, over a period of two years. Records of patients with cemented hip replacements were collected over the two-year period, by giving them a questionnaire at their follow up inspection, which happened 5-10 years after the surgery. The questionnaire was given to 50 different patients, all with cemented hip arthroplasty done before, including a wide range of demographics. Of the 50 initial prospects, 2 patients did not wish to disclose their personal information, bringing the sample number to 48 willing to participate in this study. The collected data was then cross-referenced with similar existing case studies to reach an outcome.

\section{RESULT}

During this study, a total of 48 samples were selected for collecting valid data, with a wide range of demographics, including age groups, gender, and reason for secondary revision. The man: woman ratio was $2: 3$ $(n=32: 16)$.The sample age group was divided into 4 groups based on when the surgery was done, starting from patients who were under 55 at the time of arthroplasty. A total of $58.33 \%(n=28)$ patients were under the age of 55 at the time of surgery, $16.67 \%$ $(n=8)$ were between the age group of 55 to $65.18 .75 \%$ $(n=9)$ patients were from the age group of 66 to 75 years, and the rest $6.250 \%(n=3)$ were above 75 at the time of their arthroplasty. It is to be noted that this data was heavily influenced by the average mortality rate of our country as our average life expectancy is 72.05 years. At the time of the follow-up, only $91.67 \%(n=44)$ were alive, and the remaining $8.33 \%(\mathrm{n}=4)$ were found to have passed away, of which, $6.25 \% \quad(n=3)$ had departed of natural causes, and $2.08 \%(n=1)$ died of surgery complications. The follow-up year after their initial surgery was divided into 5 groups, starting at 6 years after their surgery having a $15.91 \%(n=7)$ patients coming for secondary revision, $11.36 \%(\mathrm{n}=5)$ patients coming 7 years after the surgery, $13.64 \%(n=6)$ having followed up 8 years after the surgery, $27.27 \%(n=12)$ having followed up 9 years after the THA and the majority having done the follow up 10 years after successful surgery, at $31.82 \% \quad(n=14)$. The primary reasons for a follow-up can be divided into 3 primary groups, having the majority of patients getting follow up for resurfacing pain, at $72.73 \%(n=32), 13.64 \%(n=$ 6) for sepsis, and $13.64 \% \quad(n=6)$ coming in for dislocation.

Table-1: Sex distribution of Cemented Hip Replacements

\begin{tabular}{|l|l|l|}
\hline Sex & n & \% \\
\hline Male & 32 & $66.67 \%$ \\
\hline Female & 16 & $33.33 \%$ \\
\hline Total & 48 & 100.0 \\
\hline
\end{tabular}

Table-2: Complications in study

\begin{tabular}{|l|l|l|}
\hline Complications of study & n & \% \\
\hline Alive at follow up & 44 & $91.67 \%$ \\
\hline Deceased of natural causes & 3 & $6.25 \%$ \\
\hline Deceased of Surgery Complications & 1 & $2.08 \%$ \\
\hline Total & 48 & 100.0 \\
\hline
\end{tabular}




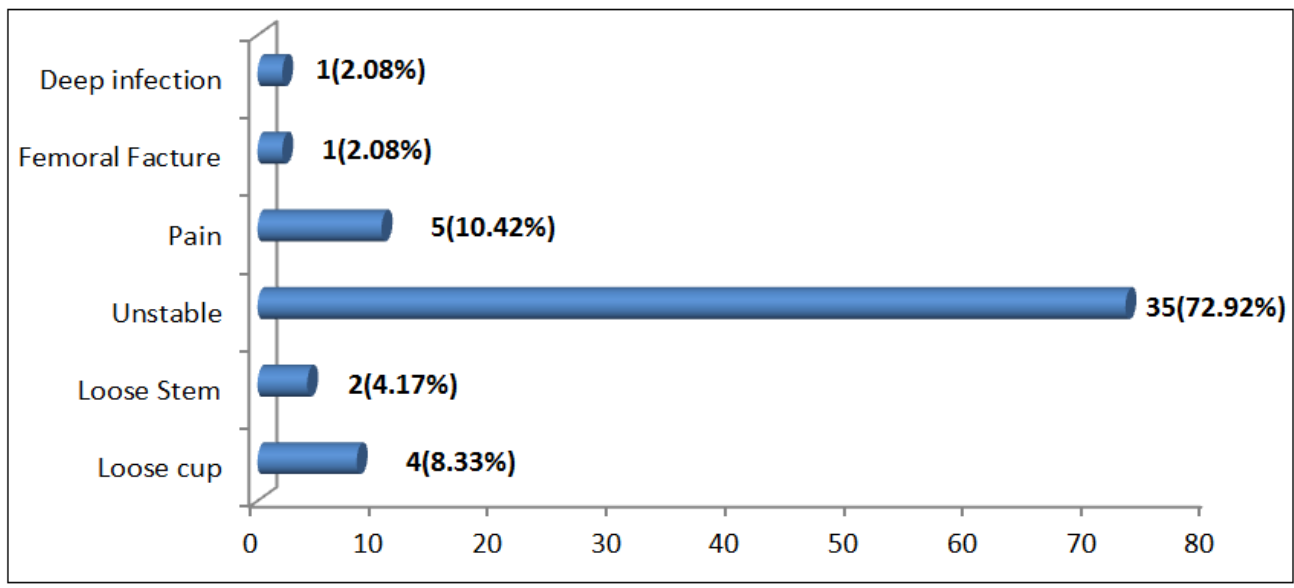

Fig-1: Reasons for hip replacement

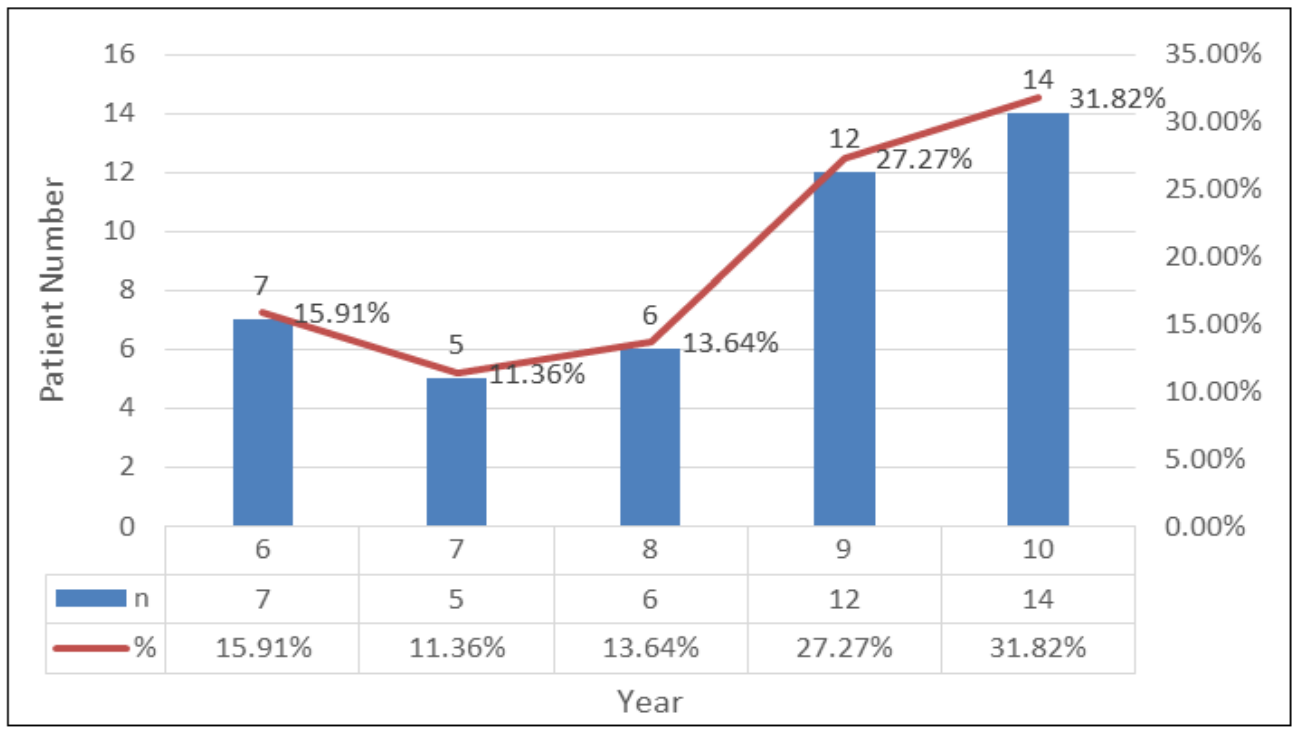

Fig-2: Follow up years of patients

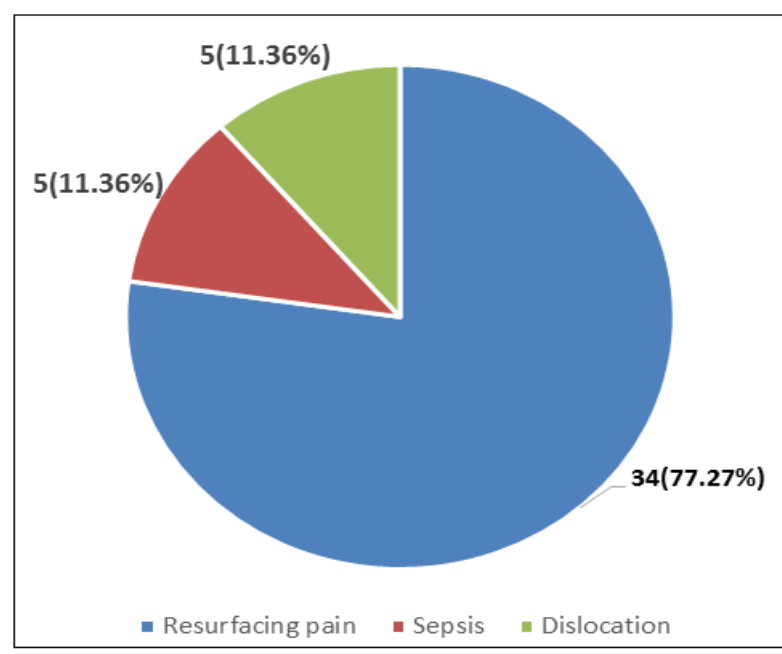

Fig-3: Outcome of Cemented Hip Replacement

\section{DisCUSSION}

For chronic joint pain due to arthritis, a patient generally opts for hip replacement surgery. This surgery can largely be categorized into two processes, Cemented hip replacement and Cementless hip replacement. This study was focused on cemented hip replacement and the evaluation of functional outcomes regarding that Cemented THA generally uses polymethylmethacrylate (PMMA) to function as a grout, producing an interlocking fit between cancellous bone and prosthesis [3]. In the case of Cemented Hip Arthroplasty, initial stability is a must for permanent fixation of acetabular cups. Biological reactions tolocalized bone resorption can often lead to implant failure. Modern fixation techniques and implants using cement have resulted in a better outcome than older cemented and historical uncemented series [4]. Cemented stems fall into two main categories: "forceclosed" and "shape-closed." Otherwise known as taperslip and composite beam respectively. Taper-slip stems, such as the Exeter stem, are collarless and have a highly polished surface finish. They achieve stability through micromotion at the prosthesis-cement interface promoting slight subsidence of the stem within the cement mantle, the generation of radial stresses, and ultimately compression at the bone-cement and prosthesis-cement interfaces. Composite beam stems, such as the later versions of the Charnley Stem, aim to 
achieve stability through a solid bond between stem, cement, and bone, maintaining the position of the stem within the mantle. Design features including rough surface finish and the presence of a collar are intended to improve stability at the prosthesis-cement interface [5]. It is generally advised to cement all femoral components of total hip replacement (THR). But two particular observations underlie this more strongly. First, it's now clear that improved cementing techniques have remarkably extended the sturdiness of the cemented femoral fixation and markedly reduced the incidence of lysis. Secondly, the incidence of femoral lysis happening around all non-cemented femoral components that are generally reported with a minimum 5-year follow-up is on the rise and it is alarming. But with good cementing in primary THR, the incidence of femoral revision for aseptic loosening at 15 to 18 years after the initial operation is merely $2 \%$ to three, even in those 50 years old and younger [6]. This has been confirmed by cross-examining a previous study regarding total hip replacements. But this does not include the overly elderly age group in the sample size, and as most of the dislocation happens in the elderly, the overall evaluation, which is the aim of this article, might differ in outcome to its cross-referenced by-parts.

Another study focused on the reasons for dislocation and lossof stems has found that the majority of the reasons are of fracture in the secondary to lysis below the tip of the stem [7]. This study focused only on one type of cemented hip replacements, specifically the matt finished femoral stems and the mode of their loosening.

It has been generally observed that the ratio of male to female coming in for hip replacements is 2 to 1 . Here, when cross-referencing with other studies performed in developed countries, it can be seen that Osteoarthritis (OA) is a leading cause of disabilityis more prevalent in women compared to men. Total joint arthroplasty is an effective treatment option for endstage OA [8]. But further analysis into those studies lead us to believe that among patients potentially at risk for the procedure, men and women within the VA system have the identical likelihood of undergoing hip arthroplasty [9]. The explanation for this disparity in countries such as ours can be explained by another study, performed via questionnaires, which shows that there's an underuse of arthroplasty for severe arthritis in both sexes, but the degree of underuse is quite 3 times as great in women as in men [10].

As our study was concluded with a limited sample size and in a country with an average life expectancy of 72.05 years, most of the complications, specifically 3 of the 4 complications found in this study, were because of death of natural causes. The remaining $2.08 \%$ of the total sample size had a complication after surgery in the form of sepsis from a fracture in theacetabulum region. This ratio of sepsis after
Cemented THR can be confirmed by another study performed by M J Spangehl [11]. When compared to the complications from cemented hip arthroplasty, the ratio of complications in cementless hip replacement can be seen as much higher, at a shockingly 9:1 ratio, with 9 being the cementless THR complications [12].

The primary analysis of the patients through the questionnaire has shown that prior to the surgery, the most prominent reason for patients to opt for a cemented THR was unstable hip joints, with almost $73 \%$ of patients having done surgery for this reason. $10 \%$ opted for THR because of pain in the coxa region, and the least reviewed reason for getting THA was a femoral fracture and deep infection in the coxa region, with both cases having one patient each. This may be influenced by the socio-economic standing of the patients, as a hip replacement can be very costly and many try to rely on medication instead of surgery for moderate to severe pain. Some of the more prominent reasons for hip arthroplasty are loose cup and loose stem, being $8.33 \%$, and $4.17 \%$ of the remaining reasons respectively.

The main reason for a follow up can be seen as resurfacing pain, as almost $3 / 4^{\text {th }}$ of the sample patients came in for their follow-up for this reason. Among other reasons, sepsis and dislocation have the same ratio. It can be theorized from this that any complications from the surgery were very limited and not severe. When cross-referencing with another study, it can be surmised that both resurfacing pain and dislocation are common after a while has passed after THR, and can happen mainly because of two reasons, both in cemented cases and cementless cases. Though further analysis has shown that cemented cases are much less likely to face these problems compared to cementless cases [13].

At the time of the surgery, more than $50 \%$ of the patients were under the age of 55, a very few over the age of 75, specifically only 3 patients opted for THR. This can also be explained by the life expectancy as the hope of survival decreases and the possible complications grow exponentially over-time after a certain age. Many after the age of 70 do not wish to take any unnecessary risk via surgery for a few more years in life. Another outlook can be that people under the age of 55 are more likely to get any major surgery done as they have a higher life expectancy and if the risks are also low compared to people over the age of 70 .

\section{Limitations OF THE STUDY}

This study faced some limitations, mostly in the case of average life expectancy. The average life expectancy in this country is 72.05 , which made it hard for patients going in for surgery to have proper followups, especially those who were over the age of 70 at the time of their operation. Another problem faced was the 
financial cost of a THR, in both cemented and cementless cases, as the surgery itself was too expensive for most patients. Many patients, despite receiving suggestions for a THR and needing it as well, did not go through with the surgery because of financial strain. Another limitation of the study was the lack of data as patients went abroad, didn't come for proper follow-ups, or had refused to share their data.

\section{CONCLUSION AND RECOMMENDATIONS}

For example $95.9 \%$ of cemented implants survived 10 ears in patients aged $\geq 75$ years compared with $93 \%$ of noncemented, $93.9 \%$ of hybrid and $93.2 \%$ of reverse hybrid. The survival of cemented replacements in patients aged 55 to 64 years was similar.

Funding: No funding sources.

Conflict of interest: None declared.

Ethical approval: The study was approved by the Institutional Ethics Committee.

\section{REFERENCES}

1. Arora M, Chan EK, Gupta S, Diwan AD. Polymethylmethacrylate bone cements and additives: A review of the literature. World journal of orthopedics. 2013 Apr 18;4(2):67.

2. Bergmann G, Deuretzbacher G, Heller M, Graichen F, Rohlmann A, Strauss J, Duda GN. Hip contact forces and gait patterns from routine activities. Journal of biomechanics. $2001 \mathrm{Jul}$ 1;34(7):859-71.

3. Maggs J, Wilson M. The relative merits of cemented and uncemented prostheses in total hip arthroplasty. Indian journal of orthopaedics. 2017 Aug;51:377-85.

4. Thanner J. The acetabular component in total hip arthroplasty: evaluation of different fixation principles. Acta Orthopaedica Scandinavica. 1999 Jan 1;70(sup286):i-41.
5. Davies N, Jackson W, Price A, Lavy C, Rees, J. FRCS Trauma and Orthopaedics Viva. Oxford University Press. 2012.

6. Harris WH. The case for cementing all femoral components in total hip replacement. Canadian Journal of Surgery. 1995 Feb 1;38(1):S55-S60.

7. Behairy YM, Harris WH. Mode of loosening of matt-finished femoral stems in primary total hip replacement. Saudi medical journal. 2002 Oct 1;23(10):1187-94.

8. Moskowitz RW (Ed.). Osteoarthritis: diagnosis and medical/surgical management. Lippincott Williams \& Wilkins. 2007.

9. Borrero S, Kent Kwoh C, Sartorius J, Ibrahim SA. Brief report: Gender and total knee/hip arthroplasty utilization rate in the VA system. Journal of general internal medicine. 2006 Mar;21(S3):S54-7.

10. Hawker GA, Wright JG, Coyte PC, Williams JI, Harvey B, Glazier R, Badley EM. Differences between men and women in the rate of use of hip and knee arthroplasty. New England Journal of Medicine. 2000 Apr 6;342(14):1016-22.

11. Spangehl MJ, Younger AS, Masri BA, Duncan CP. Diagnosis of infection following total hip arthroplasty. Instructional Course LecturesAmerican Academy of Orthopaedic Surgeons. 1998 Jan 1;47:285-96.

12. Chammout G, Muren O, Laurencikas E, Bodén H, Kelly-Pettersson P, Sjöö H, Stark A, Sköldenberg O. More complications with uncemented than cemented femoral stems in total hip replacement for displaced femoral neck fractures in the elderly: a single-blinded, randomized controlled trial with 69 patients. Acta orthopaedica. 2017 Mar 4;88(2):145-51.

13. Morshed S, Bozic KJ, Ries MD, Malchau H, Colford Jr JM. Comparison of cemented and uncemented fixation in total hip replacement: a meta-analysis. Acta Orthopaedica. 2007 Jan 1;78(3):315-26. 